\title{
Two-Dimensional Folding of Polypeptides into Molecular Nanostructures at Surfaces
}

Stephan Rauschenbach, ${ }^{*}{ }^{\dagger}$ Gordon Rinke, ${ }^{\dagger}$ Rico Gutzler, ${ }^{\dagger}$ Sabine Abb, ${ }^{\dagger}$ Alyazan Albarghash, ${ }^{\dagger}$ Duy Le, ${ }^{\dagger}$ Talat S. Rahman, ${ }^{\ddagger}$ Michael Dürr, ${ }^{\perp}$ Ludger Harnau, ${ }^{\S}$ and Klaus Kern ${ }^{\dagger, \|}$

${ }^{\dagger}$ Max Planck Institute for Solid State Research, Heisenbergstr. 1, Stuttgart DE-70569, Germany

*University of Central Florida, Orlando, Florida 32816, United States

${ }^{\perp}$ Justus Liebig University Giessen, Institute of Applied Physics, Heinrich-Buff-Ring 16, Giessen DE-35392, Germany

${ }^{\S}$ University of Stuttgart, Bernhäuserstr. 75, Leinfelden-Echterdingen DE-70771, Germany

"Ecole Polytechnique Fédérale de Lausanne, Institut de Physique, Lausanne CH-1015, Switzerland

Supporting Information

ABSTRACT: Herein we report the fabrication of molecular nanostructures on surfaces via two-dimensional (2D) folding of the nine amino acid peptide bradykinin. Soft-landing electrospray ion beam deposition in conjunction with high-resolution imaging by scanning tunneling microscopy is used to fabricate and investigate the molecular nanostructures. Subnanometer resolved images evidence the large conformational freedom of the molecules if thermal motion is inhibited and the formation of stable uniform dimers of only one specific conformation when diffusion can take place. Molecular dynamics modeling supported by density functional theory calculations give atomically precise

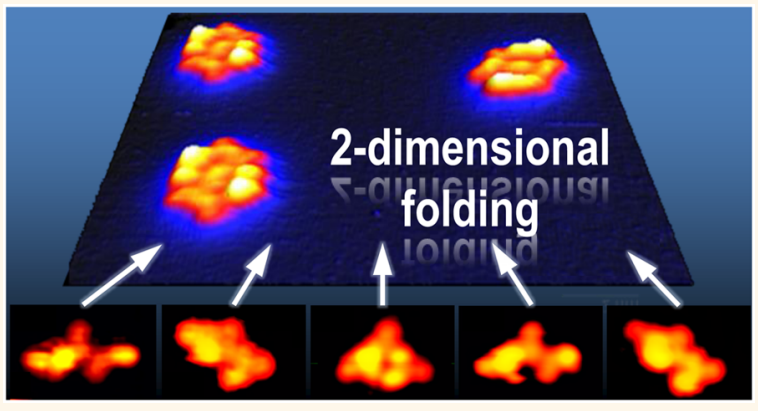
insight into the induced-fit binding scheme when the folded dimer is formed. In the absence of solvent, we find a hierarchy of binding strength from polar to nonpolar, manifested in an inverted polar-nonpolar segregation which suppresses unspecific interactions at the rim of the nanostructure. The demonstrated 2D-folding scheme resembles many key properties of its native 3D counterpart and shows that functional, molecular nanostructures on surfaces fabricated by folding could be just as versatile and specific.

KEYWORDS: peptides, surfaces, folding, scanning tunneling microscopy, electrospray ion beam deposition

I $\mathrm{n}$ protein folding, the biological self-assembly scheme that is the route to molecular functionality, a flexible amino acid (AA) sequence encodes one specific, functional conformation as well as the folding pathway to reach it within the vastness of possible conformations. ${ }^{1}$ The versatility and robustness of biological molecular functionality is based on the unique features of the process. The living cell can synthesize arbitrary sequences composed from the alphabet of 20 native AA.The folded conformation is typically densely packed and characterized by a segregation into hydrophilic and hydrophobic domains at its outside and inside, respectively. A hierarchy of covalent and various noncovalent bonds generates structural motifs that provide stability as well as an adaptable structure, enabling highly specific induced-fit binding.

Following nature's example, self-assembly of complex molecular architectures is considered a promising technology for surface functionalization, both in vacuum ${ }^{2-6}$ and at the solid-liquid interface, ${ }^{7,8}$ as demonstrated by an impressive variety of self-assembled molecular networks and nanostructures. Especially structures fabricated by vacuum sublimation of molecular building blocks onto atomically defined surfaces in ultrahigh vacuum (UHV) allow for the investigation by scanning tunneling microscopy (STM) at subnanometer resolution. ${ }^{4}$ This approach has proven to be instrumental for obtaining a thorough understanding of the complex interactions among the molecules and with the surface, because STM reveals detailed information on the atomic structure, the formation mechanism, and physical as well as chemical properties of molecular assemblies. It further bears the potential for an integration with other vacuum technologies for a controlled fabrication and analysis.

Self-assembly by molecular folding as defined above, however, has not been reported on surfaces in an artificial, vacuum environment. To be able to fold, a molecule requires a certain size to accommodate several functional sites and a flexible conformation that enables self-interaction. These properties are not only realized in natural polypeptides but

Received: September 11, 2016

Accepted: January 25, 2017

Published: January 25, 2017 
also demonstrated in various classes of synthetic, foldable molecules called foldamers, that have been synthesized. ${ }^{9}$ Because of their size and high degree of functionality, foldable molecules are not volatile. Therefore, the surface preparation by vacuum sublimation, needed for well-defined self-assembly on surfaces followed by high-resolution interrogation by STM, is not applicable. Only single $\mathrm{AAs}^{10}$ and only very few short polypeptide chains ${ }^{11,12}$ can be evaporated onto surfaces in UHV, so that their self-assembly could be studied with STM. While these examples illustrated complex molecular interactions, their size was insufficient for the observation of intramolecular interactions enabled by changes in molecular conformation, i.e., folding. Alternatively, large polymers fabricated in situ on the surface are exclusively homo- or copolymers, ${ }^{13,14}$ which lack the variety of functionality of a heteropolymer sequence that is needed for folding.

We use soft-landing electrospray ion beam deposition (ESIBD), a technique specifically useful for the gentle deposition of large nonvolatile molecules on surfaces in vacuum. ${ }^{15-18} \mathrm{We}$ recently demonstrated STM imaging of folded as well as unfolded proteins of more than a $100 \mathrm{AA}$ and the feasibility of spatial resolution at the AA level. ${ }^{19,20}$ In particular, polypeptide chains of unfolded proteins were found to be adsorbed twodimensionally (2D) in random conformations, either being immobilized or refolded $2 \mathrm{D}{ }^{19}$ While the preserved native structure occurs as a three-dimensional (3D) object on the surface, one cannot expect an unfolded polypeptide to fold into its native $3 \mathrm{D}$ structure at a surface in vacuum. ${ }^{21}$ Compared to the native environment, strength and range of inter- and intramolecular interactions of functional molecules change upon transfer to a vacuum environment. ${ }^{4}$ Additionally, an interaction of the peptide with the surface is introduced, whereas its defining interaction with the solvent is missing. ${ }^{21,22}$ This alteration is so significant that the question must be raised whether under vacuum conditions folded polypeptide structures can self-assemble with the same degree of versatility and specificity as their counterparts in the native environment. Nevertheless, the observation of folding, albeit into a random conformation, and the emergence of order in assemblies of short and medium length oligopeptides at surfaces in vacuum $^{11,12,23}$ encouraged us to search for longer sequences that show $2 \mathrm{D}$-folding into recurring, stable conformations under vacuum conditions on a surface.

Herein, we report the fabrication of molecular nanostructures via $2 \mathrm{D}$ folding of the flexible, nine AA peptide bradykinin (BK). High-resolution STM allows direct imaging of the conformational variability of the flexible molecule and the formation of one specific folded molecular nanostructure. We tune whether the molecule is kinetically trapped or is free to explore its conformational space by the choice of temperature and substrate, for which the copper surfaces $\mathrm{Cu}(110)$ and $\mathrm{Cu}(100)$ serve as model substrates that allow us to change the interaction strength. Based on the subnanometer resolved images, we perform atomistic modeling through a combination of classical molecular dynamics (MD) simulations and density functional theory (DFT) calculations.

\section{RESULTS}

Intact Deposition. BK (Figure 1a) is a nona-peptide which has been intensively studied since the discovery of its physiological effects in $1949 .{ }^{24}$ Many attempts were made to determine the structure of $\mathrm{BK}$ in different environments such as in solution by cyclic dichroism (CD) and $\mathrm{NMR}^{25}$ as well as in

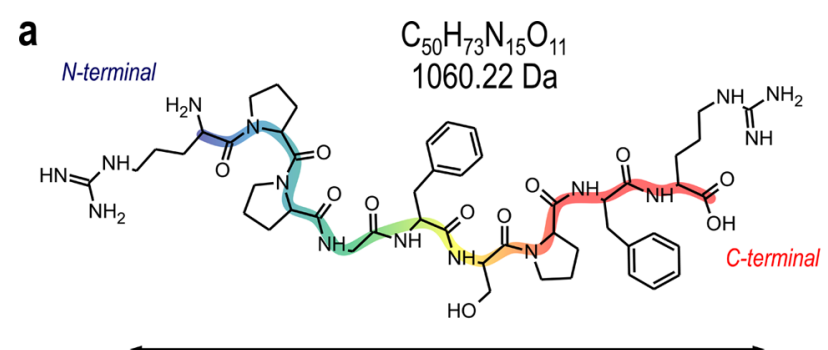

$3.6 \mathrm{~nm}$

$\mathrm{H}_{2} \mathrm{~N}$-Arg-Pro-Pro-Gly-Phe-Ser-Pro-Phe-Arg- $\mathrm{COOH}$
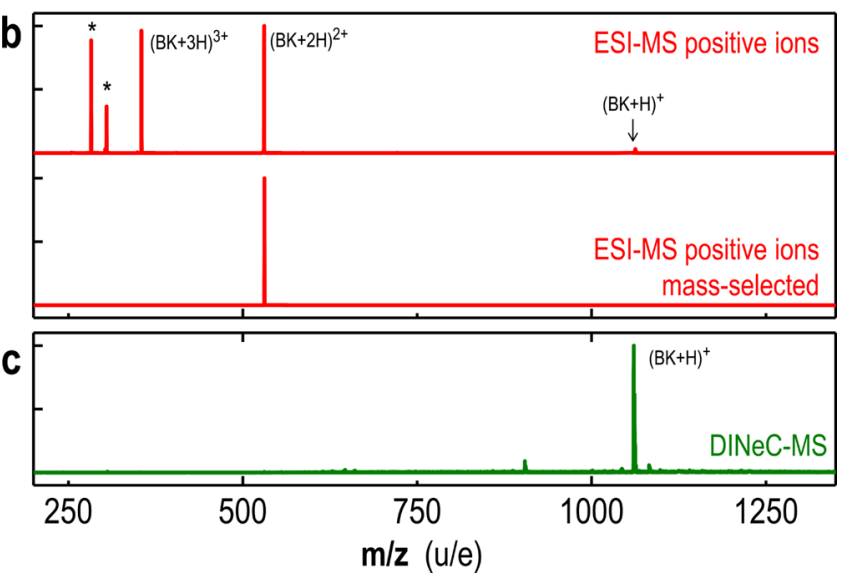

Figure 1. (a) Chemical structure and AA sequence of BK. (b) Electrospray mass spectra of BK show peaks of the molecule for the charge states $+1,+2$, and +3 . Below, the mass spectrum of the massfilter beam only contains $\mathrm{BK}+2 \mathrm{H}^{+}$ions (*marks contaminations, which are removed by $\mathrm{m} / z$ filtering). (c) Desorption ionization (DINeC) mass spectrum of BK retrieved from surfaces after softlanding. Only intact, singly protonated molecules are detected after soft landing deposition.

the gas phase by ion mobility spectroscopy ${ }^{26,27}$ or optical spectroscopy. ${ }^{28}$ In these environments the molecule's conformation is partially ambiguous: While a stable $\beta$-turn motif at the C-terminal (AA 6-9) is identified by various means (NMR, $\mathrm{CD}, \mathrm{MD})$, the $\mathrm{N}$-terminal side is an unstructured, flexible random coil, responsible for a large number of conformational states. These properties make BK a suitable model system for the investigation of the structure formation by folding on a surface.

Soft-landing electrospray ion beam deposition of massselected intact $\mathrm{BK}$ ions takes place on atomically defined surfaces in UHV at room temperature. The integrity of the molecules after deposition is ensured by mass spectrometry measurements before and after deposition. Intact $\mathrm{BK}$ is observed in the electrospray mass spectra as singly, doubly, and triply protonated ion $\mathrm{BK}+z \mathrm{H}^{+}$with $z=1-3$ (upper panel Figure $1 \mathrm{~b}$ ). For deposition, a quadrupole $\mathrm{m} / \mathrm{z}$ filter is set to transmit only the $\mathrm{BK}^{2+}$ ions, removing the other charge states and any contamination from the ion beam (lower panel in Figure $1 \mathrm{~b}$ ). On the surface, the ions neutralize very fast by losing the attached protons, most likely by electrons from the metal surface. ${ }^{29}$

After deposition, we recover the deposited material from the surface via desorption ionization induced by neutral $\mathrm{SO}_{2}$ clusters $(\mathrm{DINeC})^{30,31}$ and analyze the generated ions by mass spectrometry. The mass spectra show that exclusively intact BK 


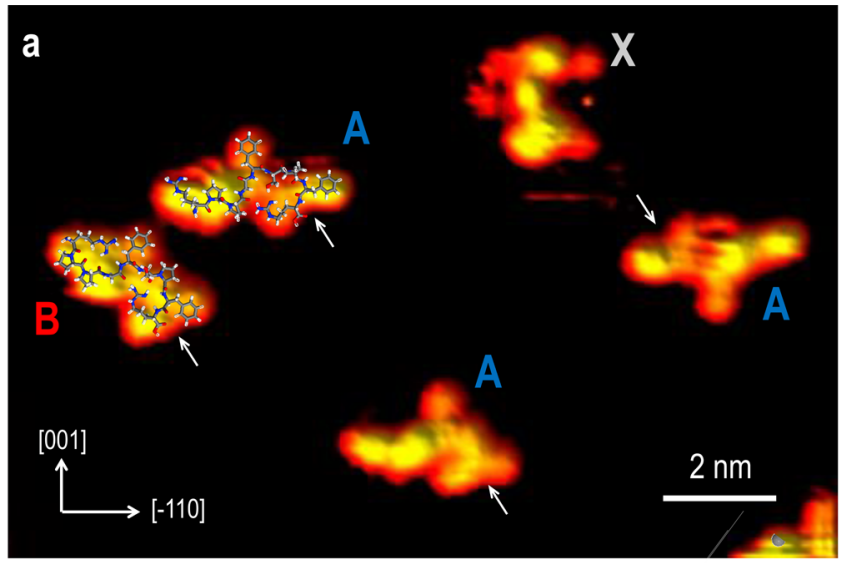

b

A

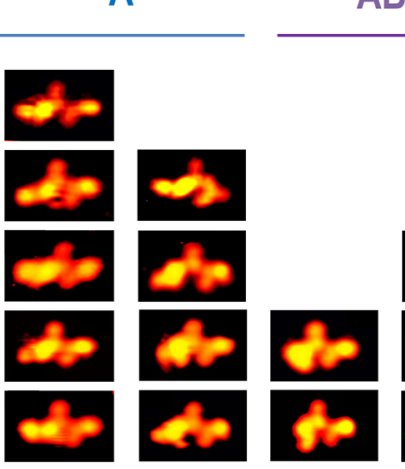

B

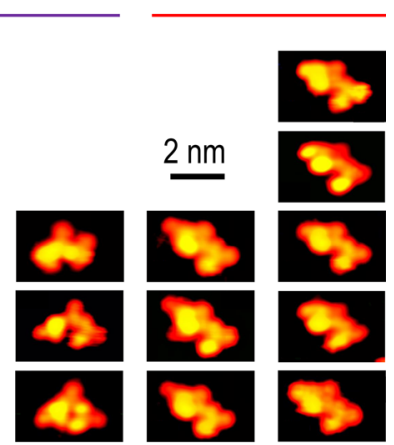

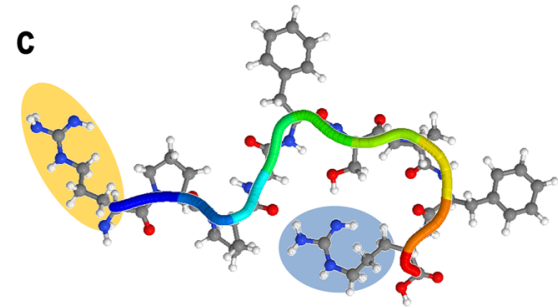

A

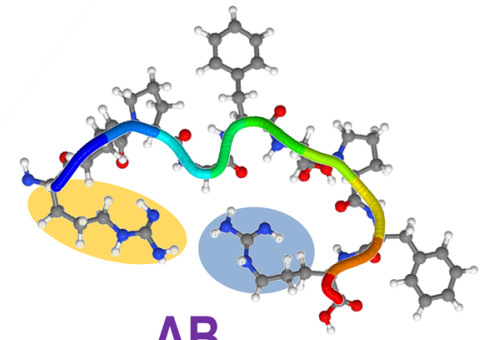

$A B$

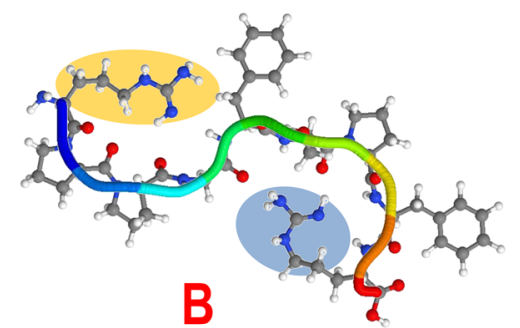

Figure 2. Conformational variability of $\mathrm{BK}$ on the $\mathrm{Cu}(110)$ surface. (a) $\mathrm{STM}$ image of several individual $\mathrm{BK}$ molecules $\left(V_{\text {gap }}=-2 \mathrm{~V}, I=50 \mathrm{pA}\right.$, $T=300 \mathrm{~K}$ ). Labels A, B, and X indicate different conformations of BK. (b) Individual BK molecules, oriented along only one symmetry direction, sorted into conformation clusters $\mathrm{A}, \mathrm{AB}, \mathrm{B}$ (left, middle, and right two columns). (c) MD model structures of characteristic conformations for each cluster.

molecules are present at the surface, which are detected as singly charged ions (Figure 1c).

Conformational Variability. On the $\mathrm{Cu}(110)$ surface, isolated individual adsorbates are imaged by STM consistent with single intact molecules (see Figure 2a). All of them exhibit similar dimensions of approximately $3 \mathrm{~nm}$ along the long axis and $1.5 \mathrm{~nm}$ along the short axis. They show three major protrusions of a height of 1.5-1.8 $\AA$ as well as several minor structural features. The adsorbates are immobilized at room temperature. A multitude of shapes is observed, indeed no pair of them is exactly the same. Structures of similar appearance are thus grouped into conformation clusters. Each conformation is found in two orientations rotated by $180^{\circ}$, which reflects the two-fold substrate symmetry and the fact that BK is an enantiopure, natural peptide, which means that no mirror images exist for a given structure. Figure $2 \mathrm{~b}$ shows individual BK molecules, aligned to one of the two symmetry directions, sorted into conformation clusters. The size of each group approximately represents the abundance of this conformation.

Two major classes of conformation can be identified. Type (A) appears as an extended strand with two short branches in the middle part at an abundance of $40 \%$. The second recurring structure (B) with an abundance of $30 \%$ appears more compact. The long axis of structure $\mathbf{A}$ is approximately aligned along the substrate's [1 $1 \overline{1} 0]$ direction. Conformation $\mathbf{B}$ is oriented close to the substrate's [111] direction. The remaining $30 \%$ of the adsorbates do not fit in either category. Some of them seem to contain elements from both major structure types (AB in Figure 2), the rest presents random, nonrecurring conformations (e.g., $\mathbf{X}$ in Figure 2a)

All observed structures of conformation A appear slightly shorter than the $3.6 \mathrm{~nm}$ expected for a fully extended BK.
Therefore, their structure cannot be explained intuitively. Certainly bends along the backbone are present, which cannot be inferred from the STM images directly. Moreover, even under the assumption of a planar conformation with only two dihedral angles for each peptide bond, already $2^{8}=256$ possible monomer conformations exist. In reality the molecular structure is much more flexible than that in the simple approximation above. For example, instead of two discrete dihedral angles, a continuous range of angles is allowed and large AA-residues such as Phe, Ser, or Arg may have conformational freedom of their own. As a result, possible conformers would differ at the angstrom level, and therefore, an intuitive interpretation even of subnanometer resolved peptide nanostructures on these surfaces is unreliable.

To shed light on the molecular structure of the observed 2Dconformations of $\mathrm{BK}$, we performed $\mathrm{MD}$ simulations that emulate the hyperthermal deposition and the ensuing structural relaxation (see the Methods section and the Supporting Information). As in the experiment, the simulations result in a large variety of molecular conformations, reproducing the large and small variations observed by STM. Figure $2 \mathrm{c}$ shows candidate structures of high abundance and stability that fit with the two most abundant conformations $\mathbf{A}$ and $\mathbf{B}$ and also some of the $\mathbf{A B}$ structures (see also overlaid structures in Figure 2a). All modeled configurations present a characteristic bend in the peptide backbone at the C-terminal side (residues Phe5 to Arg9) formed by enclosing the Arg9 residue (blue highlight in Figure 2c). The feature is stabilized by polar interactions/hydrogen bonds between the peptide backbone and Arg9. Indeed, in the STM images, the C-terminal end of all conformations appears to be similar (white arrows in Figure 2a). 

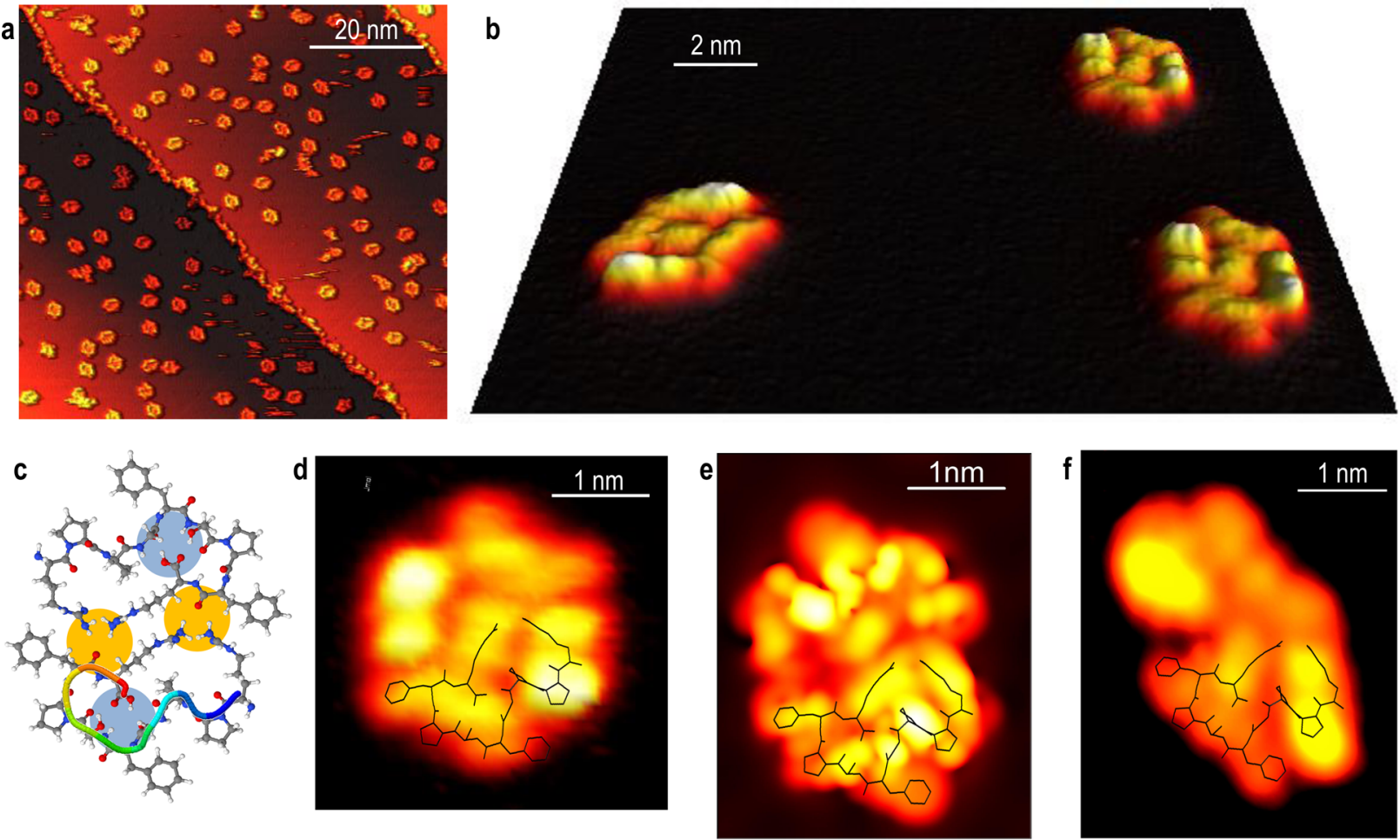

Figure 3. 2D-folded BK dimers on $\mathrm{Cu}(100)$. (a) Survey image showing many identical BK dimers at room temperature after the deposition of $\mathrm{BK}^{2+}$ ions. (b) Magnification of three dimers showing both possible orientations. (c) Model of the dimer from MD simulation. (d) Individual dimer overlaid with a MD model structure. (e) Calculated STM image of the dimer structure simulated by MD and DFT. (f) Transient dimer structure of defective conformation observed at low temperature $(40 \mathrm{~K})$.

The N-terminal side of $\mathrm{BK}$, in contrast, is significantly different for each conformation. Thereby, the position of the Arg1 residue is the defining factor: it is extended in conformer A, creating the straight, elongated impression of this conformer, while it folds inward for the more compact conformers $\mathbf{B}$ and AB, toward either Phe5 or the C-terminal, respectively (orange highlight in 2c). Our MD and DFT calculations indicate that this arrangement is complemented by the adjustment of the backbone to facilitate a strong polar interaction between Arg1 and the rest of the molecule.

Folded Dimers. The deposition of the $\mathrm{BK}^{2+}$ ion beams on the $\mathrm{Cu}(100)$ surface results in a completely different morphology of room temperature stable, compact structures of only one type. Occupying twice the area of a BK molecule on $\mathrm{Cu}(110)$, they clearly correspond to a molecular dimer (Figure $3 \mathrm{a}, \mathrm{b})$. The structure is two-fold symmetric $\left(C_{2}\right)$ and chiral. Due to the homochirality of the natural peptide $\mathrm{BK}$, only one enantiomer of such a dimer can be formed. It is observed in two orientations, rotated by $90^{\circ}$, stemming from the two-fold symmetry of the $\mathrm{Cu}(100)$ surface. The presence of only one chiral structure further suggests that one side of the dimer is strongly preferred for adsorption over the other, considering the dimer as a flat, disc-like object.

Similar to the single BK on $\mathrm{Cu}(110)$, a direct determination of the molecular structure of the BK dimer is not possible, because of its compactness. The generally uniform morphology, the observation of transient streaks in the STM images at room temperature, and kinetically trapped defective dimers at low temperature confirm the presence of mobil BK at room temperature (see also Supporting Information S-IX). We therefore assume that two $\mathrm{BK}$ molecules interact to form a dimer, which is then immobilized.
To obtain a structural model, several plausible candidate dimer structures were selected from the result of the MD simulations (see Supporting Information Figure S3). Further modeling by DFT only led to small changes in the structure, confirming the stability of the proposed structures. Due to the complexity of the molecular system and the limitation of the STM imaging to subnanometer resolution, the presented simulation scheme cannot yield a direct comparison at the atomic scale. ${ }^{32}$ We therefore focus on the comparison of the features in the STM topography simulated by DFT with the observed dimers as well as on the consistency with other available information, such as defect structures observed at low temperature.

Among the candidate structures, the configuration presented in Figure $3 c$ is the best fit to the observed dimers, found by considering all available observations. Primarily the topography, which contains information from the actual height convoluted with the local density of states (Figure 3e, see Supporting Information for details), is found to match best between experiment and theory. Usually one cannot expect exact matches, since the electronic state of the tip is unknown and the experimental images are subject to distortions such as thermal drift. However, features less affected by measurement inaccuracies, being molecular shape and height as well as defect structures, show good agreement. In particular, the general shape and the position of pronounced protrusions and depressions of the observed dimer fit better with the simulation than for the other candidate structures. Further, the presented structure is consistent with the kinetically trapped, transient structures found only at low temperatures, showing partially opened dimers (Figure 3f). These configurations would be impossible to reach with one of the other potential dimer 
structures (see Supporting Information Figure S3), confirming the arrangement of the BK molecules in the model of Figure 3c.

In the dimer, the $\mathrm{BK}$ molecule adopts a shape similar to the $\mathrm{AB}$ conformation on $\mathrm{Cu}(110)$, which is, however, more compact in comparison. At the C-terminal side, we find again a looplike feature, but it is tighter since it is stabilized by binding of the backbone with the carboxylic group of the $\mathrm{C}$ terminal (blue highlight in Figure 3c). The N-terminal tail is folded tightly inward resulting in a compact shape in which the ends of the Arg1 and Arg9 residues are in close proximity. This is the decisive feature for the dimer formation, because this site binds to an oxygen atom of the backbone of the neighboring BK molecule (orange highlight in Figure 3c).

\section{DISCUSSION}

Structure Formation. Having elucidated the molecular structure and conformation of the BK on copper surfaces with atomic precision by high-resolution STM imaging in conjunction with MD and DFT modeling, we are now able to identify motifs and discuss their relevance for molecular folding. Foremost, the folding of the peptide is enabled by the flexibility of the polypeptide backbone. However, despite having a floppy arginine side chain, the $\mathrm{N}$-terminal tail is relatively stiff, because of the presence of the double proline residues. It connects to the similarly rigid bend at the C-terminal which is enforced by intramolecular bonds. Also the C-terminal is equipped with a floppy arginine residue. Both sides of the peptide are connected by the Gly4 residue that acts as a flexible hinge due to its minimal steric demand. The rotation around this hinge defines the individual $\mathrm{BK}$ conformations on $\mathrm{Cu}(110)$ through different orientations of the $\mathrm{N}$-terminal tail and its Arg residue. Furthermore, its conformational freedom is crucial to bring the Arg residues into a position for forming the binding site with the neighboring $\mathrm{BK}$ in the dimer.

It is known from ion mobility spectroscopy measurements and from theoretical studies that the BK gas-phase ion appears in four to six preferred conformations. ${ }^{33-35}$ It was shown that the residues Ser6-Pro7-Phe8-Arg9 favor a stable $\beta$-turn motif, while the residues Arg1-Phe5 are unstructured and have no favored geometry in the gas phase. ${ }^{35,36}$ Hence, it is this flexible tail at the $\mathrm{N}$-terminus which defines the conformer in gas phase and solution.

Our observations suggest that-similar to the gas phasethe stable bend at the C-terminal and a flexible tail at the $\mathrm{N}$ terminal are the structurally defining parts of the BK molecule on the $\mathrm{Cu}(110)$ surface. However, the molecules arrive at the surface in different 3D gas-phase conformations with random orientation, angular momentum, and hyperthermal kinetic energy. Upon adsorption, they have to adopt to a quasi $2 \mathrm{D}$ conformation. The observation of recurring structures with a fixed orientation to the substrate shows that the peptide can rearrange itself and adapt to the potential energy landscape defined by the surface and its own conformational space, even in the absence of thermal-induced surface mobility at room temperature on $\mathrm{Cu}(110)$.

In particular on $\mathrm{Cu}(100)$, the vast majority of molecules is found in the same type of dimer configuration, which poses the question of the relevance of the gas-phase conformation for the final conformation on the surface. In our recent work on the adsorption of proteins from hyperthermal molecular ion beams, we have shown two effects that can give an indication: First, the native secondary structure of a protein can be retained upon adsorption, ${ }^{19}$ and second, in the absence of thermal diffusion, the adsorbed structure retains a memory of its gas-phase conformation, altered by the relaxation after hyperthermal collision. ${ }^{20}$ For the much shorter BK, this means that strong binding motifs already present in the gas-phase conformation may be preserved upon deposition. Indeed we find that the bend at the $\mathrm{C}$-terminal as well as a floppy $\mathrm{N}$-terminal tail, which are present in all gas-phase conformations, are also present similarly in the adsorbed structures. ${ }^{27}$ However, the adoption of one single conformation on $\mathrm{Cu}(100)$ indicates that dominant motifs such as the cis-trans isomerization of proline ${ }^{36}$ may be overruled by interaction with the substrate.

The height of the peptide adsorbate structures of few Angstrom is much lower than their lateral dimensions of several nanometers for individual $\mathrm{BK}$ and the dimer alike. This geometry can be described as a flat disc-like object, which infers that it has two large, flat sides, e.g., top and bottom, both allowing for adsorption. The observation of one homochiral dimer on $\mathrm{Cu}(100)$ exclusively, however, shows that one adsorption side is preferred. Our MD simulations further confirm that the same side is preferably adsorbing on $\mathrm{Cu}(110)$. The simulated structures suggest a strong adsorption of the backbone via its oxygen and nitrogen atoms, which are found in close proximity to the surface (see also Supporting Information Figure S4). Because of the steric demand of the AA residues, the above scenario is only possible in a configuration in which all bulky residues point to one side of the disc, away from the surface, or adsorb flat at the rim pointing outward. These residues also contribute to the adsorption via weaker dispersive interactions. The resulting structures are certainly strained by the surface interaction, nevertheless they are still consistent with the steric constrains of the AAs, as, for instance, given by a Ramachandran plot.

The strong interaction with the metal can be considered the reason for the quasi $2 \mathrm{D}$ arrangement of the peptide. This suggests an influence of the surface registry and/or symmetry on the formation of the peptide conformation. However, while the individual oxygen- or nitrogen-copper bond can be very strong, the lateral barrier between two adjacent $\mathrm{Cu}$ atoms is certainly much lower than the binding energy. ${ }^{4,37}$ Moreover, the single BK molecule covers an area of approximately 40 copper atoms in a flat conformation. Based on the huge number of possible $\mathrm{Cu}$ atoms to bind to and under the assumption of the low migration barriers, we can conclude from the observation of recurring molecular conformations that the substrate binding does not dominate the structure formation. Therefore, a stable folded structure will try to adopt to the substrate by finding an optimal orientation to minimize the strain within the assembly and maximize binding to the substrate. This behavior is in agreement with our observation of only one recurring structure on the weaker binding substrate $\mathrm{Cu}(100)$ and several recurring as well as random structures on strong interacting $\mathrm{Cu}(110)$. Further, we recently investigated a similar sized peptide, angiotensin II on a $\mathrm{Au}(111)$ surface, where the clearly lower substrate interaction presented itself through the absence of peptide-substrate orientation relation. ${ }^{23}$

Binding Motifs. One central paradigm of the field of molecular self-assembly at surfaces is that the mutual interaction between molecules can be defined and tuned by their design to the end of achieving desired properties such as a certain structure, hierarchical assemblies, or highly specific binding/recognition. ${ }^{38}$ This was demonstrated by employing a variety of binding types including van der Waals, hydrogen 
bonds, ${ }^{39}$ ionic bonds, ${ }^{40}$ complex coordination, ${ }^{41}$ and surface mediated interactions.

Our DFT calculations of the molecular dimer on the $\mathrm{Cu}(100)$ surface allow a view on some of these interactions at work in 2D folding. Figure 4a shows the partial charges for each

\section{a}

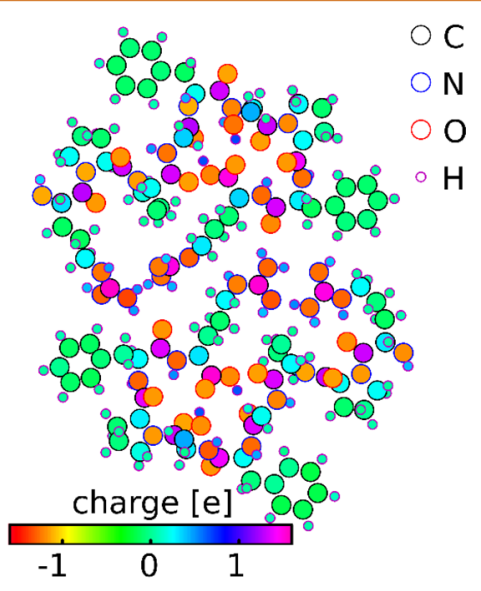

b

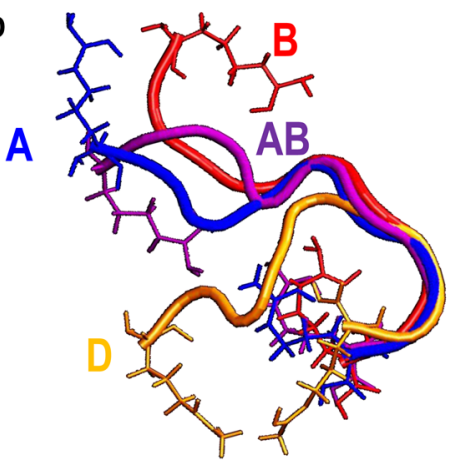

Figure 4. Results of the molecular structure simulation by MD and DFT. (a) Charge for each atom in the dimer structure. Rim color encodes the atom, and fill color the partial charge. (b) Top view of the peptide backbone with the Arg residues for $\mathrm{Cu}(110)$ conformations $\mathrm{A}, \mathrm{AB}$, and $\mathrm{B}$ and for the $\mathrm{Cu}(100)$ dimer conformation (D).

atom (filling color of the circle) calculated for the DFT-relaxed BK-dimer from the MD simulations. This visualization highlights that the intermolecular interaction in the dimer is stabilized by segregation of polar and nonpolar groups within the assembly, with mostly polar functional groups located inside the dimer, while at the outside mostly nonpolar groups are found. At the center of the structure, thus, residues are in close proximity that can form polar, noncovalent bonds. The formation of hydrogen bonds at the inside the dimer, for instance, is indicated by the positive charge calculated for the involved hydrogen atoms (e.g., C-terminal-Ser6/Arg9, Arg1Arg9-backbone).

Overall, the polar-nonpolar segregation passivates the dimers rim, shielding it from interference by other mobile molecules and thus protects the specific interaction of the two BK molecules. This conclusion is supported by the fact that single molecules are not found to interact with the dimers nor do the dimers interact with each other; only isolated dimers are observed (see Figure $3 \mathrm{a}$ and Supporting Information Figure S5).

Interestingly, it is the absence of water as a solvent that leads to an inverted behavior in the segregation of polar and nonpolar groups, as compared to a solvated $3 \mathrm{D}$ protein or peptide. In aqueous solution, proteins tend to fold nonpolar groups to the inside, gaining energy by avoiding their solvation. $^{22}$ In the absence of water, the hierarchy of intramolecular bonds, as compared to the native environment, is altered toward an emphasis of the polar interactions, because they are not screened by surrounding solvent dipoles. This we also observed after the deposition of amphiphilic surfactant molecules on surfaces in vacuum, upon which inverted membranes were formed, ${ }^{42}$ or recently for a rigid, nonfolding peptide building block, forming highly ordered hexagonal networks. $^{23}$

The hydrophilic/hydrophobic inverted folding we observe here produces decisively different structures than the folding of polypeptides in a native environment. At the same time, we evidence similarities such as conformational freedom, polarnonpolar segregation, and the formation of recurring, compact structures just as in 3D protein folding. Therefore, the question follows whether the 2D folded structures can have the specificity and, at the same time, the versatility of polypeptides in nature, ultimately allowing for tuning molecular functionality.

A hint toward this may be obtained by considering the formation mechanism of $\mathrm{BK}$ dimers on $\mathrm{Cu}(100)$. The bond between the two molecules must be highly selective, since only one stable conformation is observed at room temperature, while the presence of mobile species is indicated by streaks in the STM images (see Supporting Information). At low temperature, these mobile molecules are immobilized and can be observed in the STM as single molecules of BK of random shape. Hence the conformation of $\mathrm{BK}$ in the dimer is not defined by the interaction with the surface, but rather the result of the mutual interaction of the BK molecules stabilizing the dimer as a compactly folded entity. The comparison of the backbones (Figure $4 \mathrm{~b}$ ) for the single molecule conformations $\mathbf{A}, \mathbf{B}$, and $\mathbf{A B}$ with the dimer conformation $\mathbf{D}$ shows not only that the position of the $\mathrm{N}$-terminal tail is significantly different but also that the turn at the C-terminal is nested much tighter. Most importantly, the orientation of the two Arginine residues (Arg1 and Arg9) is the defining feature of the dimer. Together they form a binding site that connects the two BK molecules (see also Figure 3c). The structural adoption from single molecule conformation to the dimer conformation on $\mathrm{Cu}(100)$ can thus evidence an induced fit binding, a pivotal feature making protein functionality specific in the native environment.

\section{CONCLUSIONS}

The great potential of polypeptide folding lies in the ability to encode the folding pathway and thus the final conformation in the sequence via a universal synthesis approach. The folded state of a natural protein is a result of a delicate balance of a number of intramolecular interactions: hydrogen bonds, van der Waals bonds, steric interactions preferring specific binding angles, electrostatic interaction between charged AA residues, dipolar interactions, and, last but not the least, a contribution from entropy based on the hydrophilic-hydrophobic interactions. ${ }^{1,22}$ The chemical diversity of the noncovalent interactions of molecules at a surface in vacuum suggests that the folding of peptides into complex nanostructures is possible also in this environment, despite the fact that it differs significantly because of the presence of the surface and the absence of a solvent.

The formation of folded structures from BK on copper surfaces lead to nanostructures with interesting parallels to 
folded proteins: Both, despite a large conformational space, reach a ground state which is compact and in which polar and nonpolar moieties are segregated. Also, we observed conformational freedom of the molecules and specific, induced fit binding. Together with our recent work showing sequence controlled, 2D self-assembly on surfaces tuned by the polypeptide sequence, this suggests that folding at surfaces may be a promising path to sequence-programmable functional nanostructures. With ES-IBD and STM, the tools are available to prepare and analyze the folded structures at the submolecular level and to use this data in conjunction with molecular DFT and MD simulations to generate a profound understanding of the formation of folded $2 \mathrm{D}$ peptide nanostructures and their functionality.

\section{METHODS}

Bradykinin (BK, Sigma-Aldrich, B3259) is brought to the surfaces of copper single crystals by soft landing ES-IBD in ultrahigh vacuum $\left(10^{-10} \mathrm{mbar}, \mathrm{UHV}\right)$. BK is dissolved in a water/methanol mixture with $5 \%$ formic acid added at a concentration of $10^{-4} \mathrm{~mol} / \mathrm{L}$. Positively charged gas-phase ions are created from a nano electrospray at $2-4 \mathrm{kV}$ at an analyte flow rate of $15 \mu \mathrm{L} / \mathrm{h}$. The ions are transferred into a vacuum $^{43}$ and guided through six differentially pumped vacuum stages to the substrate in UHV.

Before deposition, the ion beam is thoroughly characterized. The time-of-flight mass spectrum (TOF-MS) of the positively charged BK ion beam in Figure $1 \mathrm{~b}$ is very clean and shows intense peaks between $200 \mathrm{u} / \mathrm{e}$ and $600 \mathrm{u} / \mathrm{e}$. Among them, the doubly protonated species at $531 \mathrm{u} / \mathrm{e}$ and the triply charged molecule at $354 \mathrm{u} / \mathrm{e}$ are observed with the highest intensity. The peaks marked with a star are unidentified contaminants or fragments. In addition, a peak of low intensity is found at $1061 \mathrm{u} / \mathrm{e}$, corresponding to the intact, singly protonated peptide.

In order to have one defined molecular species on the surface, the ion beam is $m / z$-filtered, excluding large clusters (which may not be detected in the mass spectrum) as well as low mass contaminants. The doubly charged molecule at $531 \mathrm{u} / \mathrm{e}$ is chosen for the deposition due to the highest intensity (see Figure $1 \mathrm{~b}$ ).

For soft-landing, the ions were decelerated to $5 \mathrm{eV} / \mathrm{z}$ via a positive voltage applied to the sample, resulting in a landing energy of $10 \mathrm{eV}$. At a flux of several hundred pA, a charge of $20-30 \mathrm{pAh}$ was deposited in few minutes. The intact deposition is confirmed by desorption ionization induced by neutral $\mathrm{SO}_{2}$ clusters (DINeC) ${ }^{30,31}$ mass spectrometry performed on reference samples after ES-IBD under the same conditions as used for the UHV deposition.

Before deposition, the $\mathrm{Cu}(100)$ and $\mathrm{Cu}(110)$ crystals are cleaned by several sputter $\left(1 \mathrm{keV} \mathrm{Ar}^{+}, 10-15 \mu \mathrm{A}, 20 \mathrm{~min}\right)$ and annealing $(750-$ $850 \mathrm{~K}, 5-10 \mathrm{~min}$ ) cycles to ensure flat, atomically clean surfaces. Directly after the deposition, the sample was transferred in situ to a variable-temperature STM (Omicron VT-STM, Omicron Nanotechnology $\mathrm{GmbH}$, Germany) and investigated at room temperature or at low temperature of $45 \mathrm{~K}$.

In order to shed light on the conformations of the observed BK monomers and dimers, we performed MD simulations of the molecule on the different surfaces using the GROMACS software package (see Supporting Information and ref 44 and references therein). The adsorption of the doubly protonated $\mathrm{BK}$ was simulated on the two copper surfaces $\mathrm{Cu}(100)$ and $\mathrm{Cu}(111)$, whose atoms were fixed in their crystallographic positions. Upon landing, the molecules neutralize by deprotonation and were relaxed resulting in stable adsorption conformations.

DFT simulations were performed for the case of the BK dimer on $\mathrm{Cu}(100)$, for which we used the conformations suggested by $\mathrm{MD}$ simulations as input for further ionic relaxation using DFT, subsequent calculation of topological STM images, and evaluation of atomic charges, which further led to characterization of the polar and nonpolar regions inside the dimer.

\section{ASSOCIATED CONTENT}

\section{Supporting Information}

The Supporting Information is available free of charge on the ACS Publications website at DOI: 10.1021/acsnano.6b06145.

Detailed description of the methods and additional details on the data analysis and results (PDF)

\section{AUTHOR INFORMATION}

\section{Corresponding Author}

*E-mail: s.rauschenbach@fkf.mpg.de.

ORCID

Stephan Rauschenbach: 0000-0002-7099-5424

Notes

The authors declare no competing financial interest.

\section{ACKNOWLEDGMENTS}

The DFT calculations of D.L. and T.S.R. was supported in part by U.S. NSF grant CHE-1310327.

\section{REFERENCES}

(1) Dill, K. A.; MacCallum, J. L. The Protein-Folding Problem, 50 Years On. Science 2012, 338, 1042-1046.

(2) Rosei, F.; Schunack, M.; Naitoh, Y.; Jiang, P.; Gourdon, A.; Laegsgaard, E.; Stensgaard, I.; Joachim, C.; Besenbacher, F. Properties of Large Organic Molecules on Metal Surfaces. Prog. Surf. Sci. 2003, $71,95-146$.

(3) Barth, J. V.; Costantini, G.; Kern, K. Engineering Atomic and Molecular Nanostructures at Surfaces. Nature 2005, 437, 671-679.

(4) Barth, J. V. Molecular Architectonic on Metal Surfaces. Annu. Rev. Phys. Chem. 2007, 58, 375-407.

(5) Bartels, L. Tailoring Molecular Layers at Metal Surfaces. Nat. Chem. 2010, 2, 87-95.

(6) Slater, A. G.; Beton, P. H.; Champness, N. R. Two-dimensional Supramolecular Chemistry on Surfaces. Chem. Sci. 2011, 2, 14401448.

(7) De Feyter, S.; De Schryver, F. Two-dimensional Supramolecular Self-assembly Probed by Scanning Tunneling Microscopy. Chem. Soc. Rev. 2003, 32, 139-150.

(8) Yang, Y.; Wang, C. Single-molecule Studies on Individual Peptides and Peptide Assemblies on Surfaces. Philos. Trans. R. Soc., A 2013, 371, 20120311.

(9) Xu, L.-P.; Liu, Y.; Zhang, X. Interfacial Self-assembly of Amino Acids and Peptides: Scanning Tunneling Microscopy Investigation. Nanoscale 2011, 3, 4901-4915.

(10) Schiffrin, A.; Riemann, A.; Auwärter, W.; Pennec, Y.; WeberBargioni, A.; Cvetko, D.; Cossaro, A.; Morgante, A.; Barth, J. V. Zwitterionic Self-assembly of L-methionine Nanogratings on the $\mathrm{Ag}(111)$ Surface. Proc. Natl. Acad. Sci. U. S. A. 2007, 104, 5279-5284.

(11) Lingenfelder, M.; Tomba, G.; Costantini, G.; Ciacchi, L. C.; De Vita, A.; Kern, K. Tracking the Chiral Recognition of Adsorbed Dipeptides at the Single-molecule Level. Angew. Chem., Int. Ed. 2007, 46, 4492-4495.

(12) Kalashnyk, N.; Nielsen, J. T.; Nielsen, E. H.; Skrydstrup, T.; Otzen, D. E.; Lgsgaard, E.; Wang, C.; Besenbacher, F.; Nielsen, N. C.; Linderoth, T. R. Scanning Tunneling Microscopy Reveals SingleMolecule Insights into the Self-Assembly of Amyloid Fibrils. ACS Nano 2012, 6, 6882-6889.

(13) Lafferentz, L.; Ample, F.; Yu, H.; Hecht, S.; Joachim, C.; Grill, L. Conductance of a Single Conjugated Polymer as a Continuous Function of Its Length. Science 2009, 323, 1193-1197.

(14) Lafferentz, L.; Eberhardt, V.; Dri, C.; Africh, C.; Comelli, G.; Esch, F.; Hecht, S.; Grill, L. Controlling On-surface Polymerization By Hierarchical And Substrate-directed Growth. Nat. Chem. 2012, 4, 215-220. 
(15) Rauschenbach, S.; Ternes, M.; Harnau, L.; Kern, K. Mass Spectrometry as a Preparative Tool for the Surface Science of Large Molecules. Annu. Rev. Anal. Chem. 2016, 9, 473-498.

(16) Johnson, G. E.; Gunaratne, D.; Laskin, J. Soft-and Reactive Landing of Ions onto Surfaces: Concepts and Applications. Mass Spectrom. Rev. 2016, 35, 439-479.

(17) Cyriac, J.; Pradeep, T.; Kang, H.; Souda, R; Cooks, R. G. LowEnergy Ionic Collisions at Molecular Solids. Chem. Rev. 2012, 112, $5356-5411$.

(18) Verbeck, G.; Hoffmann, W.; Walton, B. Soft-landing Preparative Mass Spectrometry. Analyst 2012, 137, 4393-4407.

(19) Deng, Z.; Thontasen, N.; Malinowski, N.; Rinke, G.; Harnau, L.; Rauschenbach, S.; Kern, K. A Close Look at Proteins: Submolecular Resolution of Two- and Three-Dimensionally Folded Cytochrome $\mathrm{c}$ at Surfaces. Nano Lett. 2012, 12, 2452-2458.

(20) Rinke, G.; Rauschenbach, S.; Harnau, L.; Albarghash, A.; Pauly, M.; Kern, K. Active Control of Protein Conformation on Metals by Hyperthermal Surface Interaction. Nano Lett. 2014, 14, 5609-5615.

(21) Wolynes, P. G. Biomolecular Folding in Vacuo. Proc. Natl. Acad. Sci. U. S. A. 1995, 92, 2426-2427.

(22) Chandler, D. Interfaces and the Driving Force of Hydrophobic Assembly. Nature 2005, 437, 640-647.

(23) Abb, S.; Harnau, L.; Gutzler, R.; Rauschenbach, S.; Kern, K. Two-dimensional Honeycomb Network through Sequence-controlled Self-assembly of Oligopeptides. Nat. Commun. 2016, 7, 10335.

(24) Rocha e Silva, M.; Beraldo, W. T.; Rosenfeld, G. Bradykinin, a Hypotensive and Smooth Muscle Stimulating Factor Released from Plasma Globulin by Snake Venoms and by Trypsin. Am. J. Physiol. 1949, 156, 261-273.

(25) Cann, J. R.; Liu, X.; Stewart, J. M.; Gera, L.; Kotovych, G. A CDP100 and an NMR Study of Multiple Bradykinin Conformations in Aqueous Trifluoroethanol Solutions. Biopolymers 1994, 34, 869-878.

(26) Wyttenbach, T.; von Helden, G.; Bowers, M. T. Gas-phase Conformation of Biological Molecules: Bradykinin. J. Am. Chem. Soc. 1996, 118, 8355-8364.

(27) Pierson, N. A.; Chen, L.; Valentine, S. J.; Russell, D. H.; Clemmer, D. E. Number of Solution States of Bradykinin from Ion Mobility and Mass Spectrometry Measurements. J. Am. Chem. Soc. 2011, 133, 13810-13813.

(28) Voronina, L.; Rizzo, T. R. Spectroscopic Studies of Kinetically Trapped Conformations in the Gas Phase: The Case of Triply Protonated Bradykinin. Phys. Chem. Chem. Phys. 2015, 17, 2582825836.

(29) Longchamp, J.-N.; Rauschenbach, S.; Abb, S.; Escher, C.; Latychevskaia, T.; Kern, K.; Fink, H.-W. Imaging Proteins at the Single Molecule Level. Proc. Natl. Acad. Sci. U. S. A. 2017, 201614519.

(30) Gebhardt, C. R.; Tomsic, A.; Schröder, H.; Dürr, M.; Kompa, K. L. Matrix-Free Formation of Gas-Phase Biomolecular Ions by Soft Cluster-Induced Desorption. Angew. Chem., Int. Ed. 2009, 48, 41624165.

(31) Baur, M.; Gebhardt, C. R.; Dürr, M. Desorption/ionization Induced by Neutral Cluster Impact As a Soft and Efficient Ionization Source for Ion Trap Mass Spectrometry of Biomolecules. Rapid Commun. Mass Spectrom. 2014, 28, 290-296.

(32) Schön, J. C.; Oligschleger, C.; Cortes, J. Prediction and Clarification of Structures of (Bio) Molecules on Surfaces. Z. Naturforsch., B: J. Chem. Sci. 2016, 71, 351-374.

(33) Purves, R. W.; Barnett, D. A.; Ells, B.; Guevremont, R. Gasphase Conformers of the $[\mathrm{M}+2 \mathrm{H}] 2+$ Ion of Bradykinin Investigated by Combining High-field Asymmetric Waveform Ion Mobility Spectrometry, Hydrogen/deuterium Exchange, and Energy-loss Measurements. Rapid Commun. Mass Spectrom. 2001, 15, 1453-1456.

(34) Shvartsburg, A. A.; Li, F.; Tang, K.; Smith, R. D. HighResolution Field Asymmetric Waveform Ion Mobility Spectrometry Using New Planar Geometry Analyzers. Anal. Chem. 2006, 78, 37063714.

(35) Rodriquez, C. F.; Orlova, G.; Guo, Y.; Li, X.; Siu, C.-K.; Hopkinson, A. C.; Siu, K. W. M. Gaseous Bradykinin and Its Singly,
Doubly, and Triply Protonated Forms: A First-Principles Study. J. Phys. Chem. B 2006, 110, 7528-7537.

(36) Pierson, N. A.; Chen, L.; Russell, D. H.; Clemmer, D. E. CisTrans Isomerizations of Proline Residues Are Key to Bradykinin Conformations. J. Am. Chem. Soc. 2013, 135, 3186-3192.

(37) Ternes, M.; Lutz, C. P.; Hirjibehedin, C. F.; Giessibl, F. J.; Heinrich, A. J. The Force Needed to Move an Atom on a Surface. Science 2008, 319, 1066-1069.

(38) Barth, J. V. Molecular Architectonic on Metal Surfaces. Annu. Rev. Phys. Chem. 2007, 58, 375-407.

(39) Barth, J. V.; Weckesser, J.; Cai, C.; Günter, P.; Bürgi, L.; Jeandupeux, O.; Kern, K. Building Supramolecular Nanostructures at Surfaces by Hydrogen Bonding. Angew. Chem., Int. Ed. 2000, 39, $1230-1233$.

(40) Stepanow, S.; Ohmann, R.; Leroy, F.; Lin, N.; Strunskus, T.; Wöll, C.; Kern, K. Rational Design of Two-Dimensional Nanoscale Networks by Electrostatic Interactions at Surfaces. ACS Nano 2010, 4, 1813-1820.

(41) Stepanow, S.; Lingenfelder, M.; Dmitriev, A.; Spillmann, H.; Delvigne, E.; Lin, N.; Deng, X.; Cai, C.; Barth, J.; Kern, K. Steering Molecular Organization And Host-guest Interactions Using Twodimensional Nanoporous Coordination Systems. Nat. Mater. 2004, 3, 229.

(42) Rauschenbach, S.; Rinke, G.; Malinowski, N.; Weitz, R. T.; Dinnebier, R.; Thontasen, N.; Deng, Z.; Lutz, T.; de Almeida Rollo, P. M.; Costantini, G.; Harnau, L.; Kern, K. Crystalline Inverted Membranes Grown on Surfaces by Electrospray Ion Beam Deposition in Vacuum. Adv. Mater. 2012, 24, 2761-2767.

(43) Pauly, M.; Sroka, M.; Reiss, J.; Rinke, G.; Albarghash, A.; Vogelgesang, R.; Hahne, H.; Kuster, B.; Sesterhenn, J.; Kern, K.; Rauschenbach, S. A Hydrodynamically Optimized Nano-electrospray Ionization Source and Vacuum Interface. Analyst 2014, 139, 18561867.

(44) van der Spoel, D.; Hess, B. GROMACS-the road ahead. WIREs Comput. Mol. Sci. 2011, 1, 710-715. 